\title{
Evaluation of the ability of erythromycin-resistant and -susceptible pharyngeal group A Streptococcus isolates from Spain to enter and persist in human keratinocytes
}

\section{Correspondence \\ Sebastián Albertí \\ sebastian.alberti@uib.es}

Received 1 February 2007

Accepted 28 June 2007

\author{
Maria A. Oliver, ${ }^{1}$ César García-Rey, ${ }^{2}$ Rafael Bosch ${ }^{3}$ and Sebastián Albertí ${ }^{1}$ \\ ${ }^{1}$ Institut Universitari d'Investigacions en Ciències de la Salut (IUNICS), Universitat de les Illes \\ Balears (UIB), Palma de Mallorca, Spain \\ ${ }^{2}$ Departamento Médico, GlaxoSmithKline SA, Tres Cantos, Madrid, Spain \\ ${ }^{3}$ Área de Microbiología, Departamento de Biologia, Universitat de les Illes Balears (UIB), Palma de \\ Mallorca, Spain
}

\begin{abstract}
The macrolide-resistance rate among group A Streptococcus (GAS) isolates has increased in some European countries. To investigate the reasons for this increase, the ability of 60 erythromycin-resistant and 61 erythromycin-susceptible, genetically unrelated, pharyngeal GAS isolates from Spain to enter and persist within human keratinocytes was evaluated. It was observed that erythromycin resistance was associated with the presence of prtF1, a gene related to invasiveness, whereas no association was observed with the ability to enter human keratinocytes. However, the ability to enter human keratinocytes was not associated with the presence of prtF1 or with the emm type, suggesting that interaction with keratinocytes depends on several characteristics of the isolate. Almost all strains (95.9\%) were capable of persisting within human keratinocytes. However, most of them ( $91.7 \%)$ exhibited a decline in viability over time. Interestingly, the ability to persist within keratinocytes protected from the action of the $\beta$ lactams was higher among erythromycin-resistant isolates and correlated with their ability to avoid the induction of cellular lysis. These observations suggest that if the carrier state results from intracellular GAS survival, the association between erythromycin resistance and intracellular persistence may represent a serious problem for the eradication of these isolates.
\end{abstract}

\section{INTRODUCTION}

Streptococcus pyogenes or group A Streptococcus (GAS) is the most common cause of bacterial pharyngitis and colonizes up to one-half of all school-age children in nonepidemic periods. Although penicillin remains the drug of choice for the treatment of streptococcal infections, macrolide antibiotics are recommended as suitable alternatives for patients allergic to penicillin.

In the last decade, the incidence of macrolide resistance has increased dramatically in different countries and nowadays represents an important problem, particularly in southern European countries (Bingen et al., 2004; Dicuonzo et al., 2002; Melo-Cristino \& Fernandes, 1999), including Spain (Albertí et al., 2003), where in some regions the incidence is very high (62.3\%) (Pérez-Trallero et al., 2005). Resistance to macrolides is based on the presence of an active drug efflux pump encoded by mefA (M phenotype) or on target site modification by methylases encoded by

Abbreviation: GAS, group A Streptococcus.
ermB and ermA and expressed constitutively (constitutive resistance, cMLS phenotype) or due to the presence of an inducing antibiotic (inducible resistance, iMLS phenotype) (Leclercq, 2002).

It is well established that increased resistance to macrolides may be due to the extensive use of these antimicrobials. Thus the relationship between macrolide consumption and spread of resistance in GAS has been described (García-Rey et al., 2002; Pérez-Trallero, et al., 2005). However, the increased incidence of macrolide-resistant GAS isolates may also be due to the presence of certain virulence factors in these isolates that would confer advantages for colonizing the pharynx.

Attachment to and entry of GAS into epithelial cells may be considered the initial steps in pharynx infection. Several virulence factors are involved in these steps, including the M protein (Courtney et al., 2002; Schrager et al., 1998) and the fibronectin-binding protein F1 (Molinari et al., 1997; Ozeri et al., 1998). The M protein, encoded by the $\mathrm{emm}$ gene, promotes the entry of the micro-organism through 
the recognition of different receptors on the surface of the eukaryotic cells depending on the M serotype (Courtney et al., 2002). The fibronectin-binding protein F1, encoded by gene prtF1, mediates GAS adherence to cells and the extracellular matrix, and efficient cell internalization via integrins (Ozeri et al., 1998).

Facinelli et al. (2001) reported the association between erythromycin resistance in GAS and the ability to enter human respiratory cells, as demonstrated by the presence of the fibronectin-binding protein F1. This study raised a serious concern since strains combining erythromycin resistance and ability to enter human respiratory-tract cells may be able to escape treatment with both $\beta$-lactams, by virtue of intracellular location, and macrolides, by virtue of resistance. More recently, Haller et al. (2005) confirmed the association between resistance to erythromycin and the presence of the fibronectin-binding protein F1 in GAS isolates from German paediatric patients.

In this report, we characterized the ability of a large number of erythromycin-susceptible and erythromycinresistant GAS strains isolated in Spain to invade and persist in human keratinocytes to better understand the reasons for the increased frequency of erythromycin-resistant GAS in Spain.

\section{METHODS}

Bacterial strains and their characterization. Sixty erythromycinresistant (MIC $\geqslant 1 \mu \mathrm{g} \mathrm{ml}^{-1}$ ) and 61 erythromycin-susceptible (MIC $<1 \mu \mathrm{g} \mathrm{ml}^{-1}$ ) GAS strains were included in this study. They were isolated from patients with community-acquired acute pharyngitis and belonged to a large collection of isolates obtained between 1996 and 1999 at eight different hospitals from different geographical locations in Spain (Albertí et al., 2003). The genetic relatedness of the isolates was verified by emm typing and PFGE following protocols previously described (Albertí et al., 2003). In the case of identity, only one isolate was used in this study. Isolates were maintained frozen and cultured on $5 \%$ sheep blood agar plates before testing.

The MIC for erythromycin was determined according to the guidelines of the Clinical and Laboratory Standards Institute (formerly the National Committee for Clinical Laboratory Standards) (NCCLS, 2000). Erythromycin-resistant strains were differentiated into resistance phenotypes by the double-disc test (Seppala et al., 1993) and screened for the presence of ermB, ermTR, $m e f A$ and prtF1 genes as previously described (Neeman et al., 1998; Seppala et al., 1998; Sutcliffe et al., 1996).

Cell culture assays. For the invasiveness studies, we followed the protocol described by Schrager et al. (1996). Monolayers of human dysplastic oral keratinocytes [European Collection of Cell Cultures (ECACC), reference 94122104] were grown to confluence in DMEM (Sigma) supplemented with $2 \mathrm{mM}$ glutamine, $5 \mu \mathrm{g}$ hydrocortisone $\mathrm{ml}^{-1}$ and $10 \%$ fetal bovine serum plus penicillin and streptomycin in 24 -well tissue culture plates $\left(\sim 5 \times 10^{5}\right.$ cells per well $)$. Monolayers were washed three times to remove unattached cells, then inoculated with approximately $10^{5}$ c.f.u. of GAS per well. After incubation for $2 \mathrm{~h}$ at $37^{\circ} \mathrm{C}$ in $5 \% \mathrm{CO}_{2}$, the monolayers were washed with PBS and then incubated for $1 \mathrm{~h}$ with fresh medium containing gentamicin $(100 \mu \mathrm{g}$ $\mathrm{ml}^{-1}$ ) to kill extracellular bacteria. A $50 \mu \mathrm{l}$ aliquot of supernatant fluid was removed for quantitative culture to confirm killing of extracellular bacteria. To recover intracellular bacteria, the gentamicin-containing medium was removed, the keratinocytes were washed again, then lysed, and the contents of each well were vortexed for $15 \mathrm{~s}$ to evenly disperse the bacteria. Serial dilutions in water were done and an aliquot was spread on $5 \%$ sheep blood agar plates for quantitative culture. Cell-invasion efficiency was established as previously described (Facinelli et al., 2001), on the basis of the percentage of the intracellular bacteria (with reference to the initial inoculum) recovered after the incubation of infected cells with gentamicin, which is unable to enter into the cells. Depending on cell-invasion efficiency ( $>10 \%, 1-10 \%$ or $<1 \%$ ), strains were defined as highly invasive, weakly invasive or non-invasive, respectively.

For the persistence assays, we used ampicillin to kill extracellular bacteria and the incubation step was prolonged up to $18 \mathrm{~h}$. After that, the antibiotic-containing medium was washed away, the keratinocytes were lysed, and intracellular bacteria were recovered as described above. Persistence was calculated as the percentage of intracellular bacteria recovered after $18 \mathrm{~h}$ of incubation with respect to the intracellular bacteria recovered $2 \mathrm{~h}$ after inoculation. Depending on persistence $(>50 \%, 1-50 \%$ or $<1 \%)$, strains were defined as highly persistent, weakly persistent or non-persistent.

Cytotoxicity was estimated by the method described by Tsai et al. (1999). In brief, keratinocytes were infected as described above and after 3 and $18 \mathrm{~h}$ of incubation with ampicillin-containing medium this was replaced with fresh culture medium containing the dye WST1 (Boehringer Mannheim) and incubated at $37^{\circ} \mathrm{C}$ for $30 \mathrm{~min}$. The plates were read on a microplate reader and the per cent cytotoxicity was calculated with respect to uninfected monolayers (100\% viability). Depending on the cytotoxicity ( $\geqslant 90 \%$ or $<90 \%$ ), strains were defined as non-toxic or toxic, respectively.

Each streptococcal strain was tested in duplicate in three independent experiments done on different days. Results were analysed using Fisher's test and found significant when $P<0.05$.

\section{RESULTS AND DISCUSSION}

The detection of prtF1, cell-invasion efficiency and, for erythromycin-resistant strains, the resistance phenotype and genotype are shown in Table 1.

Overall, the proportion of prtF1-positive strains was higher among erythromycin-resistant strains than among erythromycin-susceptible strains $[55 / 60 \quad(91.7 \%) \quad$ vs $46 / 61$ $(75.4 \%) ; P<0.01]$. These data confirm the results obtained by Facinelli et al. (2001) in Italy and Haller et al. (2005) in Germany. However, the rate of prtF1-positive isolates, among both erythromycin-resistant and erythromycinsusceptible isolates, was markedly higher in our study than in these studies. Since most of the resistant strains included in our study exhibited the M phenotype ( $88.3 \%$ ), we could not study whether the presence of prtF1 was associated with a particular resistance phenotype ( $\mathrm{M}$ or MLS phenotype) as described by Cocuzza et al. (2004).

The efficiency of bacterial entry into keratinocytes varied widely among isolates, although the behaviour of each individual isolate was quite reproducible. Interestingly, the proportion of highly invasive isolates was higher among those that were erythromycin-resistant than among erythromycin-susceptible isolates [32/60 (53.3\%) vs $27 / 61$ $(44.3 \%) ; P=0.2]$ but the difference was not significant as 
Table 1. Characteristics of erythromycin-resistant and erythromycin-susceptible GAS strains included in this study

\begin{tabular}{|c|c|c|c|c|c|c|c|c|c|}
\hline \multirow[t]{2}{*}{ Resistance phenotype } & \multirow[t]{2}{*}{$n$} & \multicolumn{3}{|c|}{ Resistance genotype } & \multicolumn{2}{|c|}{ prtF1 gene } & \multicolumn{3}{|c|}{ Cell-invasion efficiency ${ }^{\star}$} \\
\hline & & $e r m B+m e f A$ & ermB & $m e f A$ & + & - & High & Weak & Non-invasive \\
\hline \multirow[t]{2}{*}{ All resistant } & 60 & $9(15 \%)$ & $4(6.7 \%)$ & $42(70 \%)$ & $55(91.7 \%)$ & & $32(53.3 \%)$ & $19(31.7 \%)$ & $4(6.7 \%)$ \\
\hline & & $1(1.6 \%)$ & $2(3.3 \%)$ & $2(3.3 \%)$ & & $5(8.3 \%)$ & $3(5 \%)$ & $2(3.4 \%)$ & 0 \\
\hline M & 53 & 8 & 0 & 42 & 50 & & 29 & 17 & 4 \\
\hline cMLS & & 0 & 0 & 0 & & 0 & 0 & 0 & 0 \\
\hline \multirow[t]{2}{*}{ iMLS } & 4 & 1 & 1 & 0 & 2 & & 2 & 0 & 0 \\
\hline & & 0 & 2 & 0 & & 2 & 0 & 2 & 0 \\
\hline \multirow[t]{2}{*}{ Erythromycin-susceptible } & 61 & & & & $46(75.4 \%)$ & & $27(44.3 \%)$ & $14(22.9 \%)$ & $5(8.2 \%)$ \\
\hline & & & & & & $15(24.6 \%)$ & $8(13.1 \%)$ & $6(9.8 \%)$ & $1(1.6 \%)$ \\
\hline
\end{tabular}

${ }^{*}$ Cell-invasion efficiency was established on the basis of the percentage of intracellular bacteria (with reference to the initial inoculum). Depending on cell-invasion efficiency $(>10 \%, 1-10 \%$ or $<1 \%)$, strains were defined as highly invasive, weakly invasive or non-invasive.

described by Facinelli et al. (2001). This discrepancy may have different explanations. The clonal independence of the macrolide-resistant isolates was not assessed in the original study. In fact, in a later study, Spinaci et al. (2004) reported that three major clones accounted for $47 \%$ of the erythromycin-resistant isolates included in the study of Facinelli et al. (2001). In contrast, in our study, we used genetically unrelated strains as assessed by emm-typing and PFGE. Also, in our invasion assays, we used monolayers of human keratinocytes, the major cell type of pharyngeal epithelium, which are more representative of the pharynx infection caused by GAS than the tumoral cell line A549 of type II pneumocytes used by Facinelli et al. (2001). Finally, in our study, most of the erythromycin-resistant isolates exhibited the $\mathrm{M}$ phenotype of resistance $(88.3 \%)$, while in the study of Facinelli et al. (2001), only $40.5 \%$ of the isolates exhibited this phenotype. It is likely that this difference may contribute to explaining our results, although, to date, it is unclear whether the resistance phenotype of the isolates is associated with their cellinvasiveness ability.

We did not find a direct correlation between the presence of prtF1 and the ability to invade keratinocytes, suggesting that other bacterial components are involved in the entry of GAS into these human cells. M protein, encoded by emm, is critical in causing pharyngitis (Ashbaugh et al., 2000), and may recognize different receptors on the surface of the eukaryotic cells depending on the M type (Courtney et al., 2002). We investigated whether the emm type was associated with the invasion efficiency. As shown in Table 2, we did not find a clear association between the emm type of the isolates and their ability to enter into keratinocytes. Moreover, isolates with the same emm type showed differences in their ability to enter into keratinocytes. Thus isolates expressing the prevalent emm types among the erythromycin-resistant strains from Spain (emm types
4, 12, 75 and st1815; Albertí et al., 2003) presented a wide heterogeneity in their ability to invade cells. These results are consistent with those reported by Molinari \& Chhatwal (1998), who demonstrated that the ability of GAS to adhere to and invade epithelial cells seemed to depend on the isolate, not on the $\mathrm{M}$ type. Probably other factor(s) besides the $\mathrm{M}$ protein are involved in the bacteria-cell interaction.

Table 2. Cell-invasion efficiency among GAS with different emm types

\begin{tabular}{|lrrcc|}
\hline \multirow{2}{*}{ emm type } & $\boldsymbol{n}$ & \multicolumn{3}{c|}{ Cell-invasion efficiency } \\
\cline { 3 - 5 } & & High & Weak & Non-invasive \\
\hline 1 & 6 & 1 & 4 & 1 \\
2 & 4 & 2 & 1 & 1 \\
3 & 8 & 4 & 4 & 0 \\
4 & 22 & 17 & 5 & 0 \\
6 & 6 & 6 & 0 & 0 \\
9 & 6 & 2 & 3 & 1 \\
11 & 2 & 1 & 1 & 0 \\
12 & 19 & 8 & 7 & 4 \\
13 & 3 & 2 & 0 & 1 \\
22 & 11 & 9 & 2 & 0 \\
28 & 6 & 4 & 2 & 0 \\
41 & 1 & 1 & 0 & 0 \\
44 & 3 & 0 & 3 & 0 \\
50 & 1 & 1 & 0 & 0 \\
75 & 8 & 2 & 6 & 0 \\
st1 & 16 & 10 & 5 & 1 \\
& 16 & & & \\
\hline
\end{tabular}

*Cell-invasion efficiency was established on the basis of the percentage of intracellular bacteria (with reference to the initial inoculum). Depending on cell-invasion efficiency $(>10 \%, 1-10 \%$ or $<1 \%$ ), strains were defined as highly invasive, weakly invasive or non-invasive. 
For instance, the hyaluronic acid capsule may modulate the ability of GAS to attach to and invade human keratinocytes (Schrager et al., 1998).

The biological significance of GAS entry into host cells is not well defined. Entry into epithelial cells generally leads to their lysis. However, it is not clear whether this mechanism represents a strategy of the pathogen to spread and invade deeper tissues or a host mechanism to initiate inflammation and control the infection (Cywes \& Wessels, 2001; Tsai et al., 1999). Also, entry into epithelial cells may represent a reservoir for persistent throat carriage in a site where the bacteria are protected from the actions of the immune system and antibiotics, particularly penicillin, the drug of choice in the treatment of streptococcal pharyngitis (Facinelli et al., 2001; Kaplan et al., 2006). Recently, Spinaci et al. (2006) investigated the intracellular persistence of 31 erythromycin-resistant isolates. They demonstrated that the resistant isolates were capable of persisting in A549 cells. In this study, we compared the intracellular persistence of erythromycin-resistant and erythromycinsusceptible isolates. For this purpose, we studied recovery of the intracellular bacteria from infected human keratinocytes after $18 \mathrm{~h}$ of incubation in cell monolayers in the presence of a $\beta$-lactam (ampicillin) that fails to kill intracellular GAS. Overall, the proportion of highly persistent strains was higher among erythromycin-resistant strains than among erythromycin-susceptible strains [16/60 $(26.7 \%)$ vs $7 / 61(11.4 \%) ; P=0.019]$ (Table 3). However, we recovered viable intracellular bacteria after $18 \mathrm{~h}$ of exposure to ampicillin in 111 (91.7\%) of the isolates, either resistant or susceptible to erythromycin. We found that in most of the cases $(116 ; 95.9 \%)$, intracellular bacteria were not able to multiply within keratinocytes and showed a decline in their viability over time. Interestingly, five erythromycin-resistant isolates exhibited the highest persistent rates. These data are consistent with those reported recently by Spinaci et al. (2006).

Although ampicillin cannot enter into eukaryotic cells, clearance of bacteria may be due to exposure of the microorganism to the ampicillin present in the medium since
GAS may induce apoptosis and lysis of epithelial cells (Tsai et al., 1999). To investigate this hypothesis, we determined the viability of keratinocytes after $18 \mathrm{~h}$ of exposure to intracellular bacteria. All isolates were able to reduce the viability of the human keratinocytes, suggesting that the decline of bacterial viability was due to the exposure of bacteria to extracellular ampicillin after lysis of the keratinocytes by GAS. However, we found a large heterogeneity in the ability of the isolates to induce the lysis of the cells (Table 3). Interestingly, we found an inverse correlation between the ability of the isolates to persist intracellularly and their ability to lyse the cells. Thus the isolates with a higher ability to persist within the cells were less cytotoxic than those with a lower ability [21/23 $(91.3 \%)$ vs $7 / 23$ (30.4\%), $P=0.03]$, suggesting that persistence may be associated with the ability of the isolate to remain intracellularly without the induction of cell lysis. These results are in agreement with those reported by Molinari et al. (2001), who demonstrated that intracellular persistence depends on the efficient expression of the $\mathrm{Nra}$ regulator, which decreases the expression of genes involved in host cell damage, such as those for streptolysin $S$ and SpeB (Beckert et al., 2001).

In summary, our study shows that in GAS isolates from Spain, resistance to erythromycin is associated with the presence of the prtF1 gene but not with the ability to enter human keratinocytes, which probably depends on characteristics of the isolate other than the emm type or the presence of prtF1. Interestingly, the ability to persist within keratinocytes, and therefore be protected from the action of the $\beta$-lactams, is higher among erythromycin-resistant isolates and it seems to be related to their ability to avoid the induction of cellular lysis. These observations suggest that if the state of the upper respiratory tract in GAS carriers results in intracellular GAS survival, the association between erythromycin resistance and intracellular persistence may represent a serious problem for the eradication of these isolates because they will be able to escape both $\beta$ lactams, by virtue of intracellular location, and macrolides, by virtue of resistance.

Table 3. Ability of erythromycin-resistant or erythromycin-susceptible GAS to persist in and induce keratinocyte lysis

\begin{tabular}{|c|c|c|c|c|c|}
\hline \multirow[t]{2}{*}{ Erythromycin phenotype } & \multicolumn{3}{|c|}{ Persistence $^{\star}$} & \multicolumn{2}{|c|}{ Cytotoxicity $\dagger$} \\
\hline & High & Weak & Non-persistent & Toxic & Non-toxic \\
\hline \multirow[t]{2}{*}{ Erythromycin-resistant } & $2(3.3 \%)$ & $6(10 \%)$ & $7(11.7 \%)$ & $15(25 \%)$ & 0 \\
\hline & $14(23.3 \%)$ & $28(46.7 \%)$ & $3(5 \%)$ & 0 & $45(75 \%)$ \\
\hline \multirow[t]{2}{*}{ Erythromycin-susceptible } & 0 & $16(26.2 \%)$ & $7(11.5 \%)$ & $23(37.7 \%)$ & 0 \\
\hline & $7(11.5 \%)$ & $27(44.3 \%)$ & $4(6.6 \%)$ & 0 & $38(62.3 \%)$ \\
\hline
\end{tabular}

*Persistence was calculated as the percentage of intracellular bacteria recovered after $18 \mathrm{~h}$ of incubation with respect to the intracellular bacteria recovered $2 \mathrm{~h}$ after inoculation. Depending on persistence ( $>50 \%, 1-50 \%$ or $<1 \%)$, strains were defined as highly persistent, weakly persistent or non-persistent.

$\dagger$ Cytotoxicity was calculated with respect to uninfected monolayers (100\% viability). Depending on the cytotoxicity ( $\geqslant 90 \%$ or $<90 \%)$, strains were defined as toxic or non-toxic, respectively. 


\section{ACKNOWLEDGEMENTS}

This study was supported by a grant from GlaxoSmithKline SA and by Ministerio de Sanidad y Consumo, Instituto de Salud Carlos III, Spanish Network for the Research in Infectious Diseases (REIPI RD06/0008). We thank Luis Martínez-Martínez for his comments on the manuscript.

\section{REFERENCES}

Albertí, S., Garcia-Rey, C., Domínguez, M. A., Aguilar, L., Cercenado, E., Gobernado, M., Garcia-Perea, A. \& Spanish Surveillance Group for Respiratory Pathogens (2003). Survey of emm gene sequences from pharyngeal Streptococcus pyogenes isolates collected in Spain and their relationship with erythromycin susceptibility. J Clin Microbiol 41, 2385-2390.

Ashbaugh, C. D., Moser, T. J., Shearer, M. H., White, G. L., Kennedy, R. C. \& Wessels, M. R. (2000). Bacterial determinants of persistent throat colonization and the associated immune response in a primate model of human group A streptococcal pharyngeal infection. Cell Microbiol 2, 283-292.

Beckert, S., Kreikemeyes, B. \& Podbielski, A. (2001). The group A streptococcal rofA gene is involved in the control of several virulence genes and eukaryotic cell attachment and internalization. Infect Immun 69, 534-537.

Bingen, E., Bidet, P., Mihaila-Amrouche, L., Doit, C., Forcet, S., Brahimi, N., Bouvet, A. \& Cohen, R. (2004). Emergence of macrolideresistant Streptococcus pyogenes strains in French children. Antimicrob Agents Chemother 48, 3559-3562.

Cocuzza, C. E., Lanzafame, A., Sisto, F., Broccolo, F. \& Mattina, R. (2004). Prevalence of the internalization-associated gene prtF1 in a bacterial population of Streptococcus pyogenes isolated from children with acute pharyngotonsillitis before and after antibiotic therapy. Microb Drug Resist 10, 264-268.

Courtney, H. S., Hasty, D. L. \& Dale, J. B. (2002). Molecular mechanisms of adhesion, colonization and invasion of group A streptococci. Ann Med 34, 77-87.

Cywes, C. \& Wessels, M. R. (2001). Group A streptococcus tissue invasion by CD44-mediated cell signalling. Nature 414, 648-652.

Dicuonzo, G., Fiscarelli, E., Gherardi, G., Lorino, G., Battistoni, F., Landi, S., De Cesaris, M., Petitti, T. \& Beall, B. (2002). Erythromycinresistant pharyngeal isolates of Streptococcus pyogenes recovered in Italy. Antimicrob Agents Chemother 46, 3987-3990.

Facinelli, B., Spinaci, C., Magi, G., Giovanetti, E. \& Varaldo, P. E. (2001). Association between erythromycin resistance and ability to enter human respiratory cells in group A streptococci. Lancet 358, 3033.

Garcia-Rey, C., Aguilar, L., Baquero, F., Casal, J. \& Martin, J. E. (2002). Pharmacoepidemiological analysis of provincial differences between consumption of macrolides and rates of erythromycin resistance among Streptococcus pyogenes isolates in Spain. J Clin Microbiol 40, 2959-2963.

Haller, M., Fluegge, K., Arri, A. J., Adams, B. \& Berner, R. (2005). Association between resistance to erythromycin and the presence of the fibronectin binding protein $\mathrm{F} 1$ gene, prtF1, in Streptococcus pyogenes isolates from German pediatric patients. Antimicrob Agents Chemother 49, 2990-2993.

Kaplan, E. L., Chhatwal, G. S. \& Rohde, M. (2006). Reduced ability of penicillin to eradicate ingested group A streptococci from epithelial cells: clinical and pathogenetic implications. Clin Infect Dis 43, 13981406.
Leclercq, R. (2002). Mechanisms of resistance to macrolides and lincosamides: nature of the resistance elements and their clinical implications. Clin Infect Dis 34, 482-492.

Melo-Cristino, J. \& Fernandes, M. L. (1999). Streptococcus pyogenes isolated in Portugal: macrolide resistance phenotypes and correlation with T types. Microb Drug Resist 5, 219-225.

Molinari, G. \& Chhatwal, G. S. (1998). Invasion and survival of Streptococcus pyogenes in eukaryotic cells correlates with the source of the clinical isolates. J Infect Dis 177, 1600-1607.

Molinari, G., Talay, S. R., Valentin-Weigand, P., Rohde, M. \& Chhatwal, G. S. (1997). The fibronectin-binding protein of Streptococcus pyogenes, SfbI, is involved in the internalization of group A streptococci by epithelial cells. Infect Immun 65, 1357-1363.

Molinari, G., Rohde, M., Talay, S. R., Chhatwal, G. S., Beckert, S. \& Podbielski, A. (2001). The role played by the group A streptococcal negative regulator Nra on bacterial interactions with epithelial cells. Mol Microbiol 40, 99-114.

NCCLS (2000). Methods for Dilution Antimicrobial Susceptibility Tests for Bacteria that Grow Aerobically, 5th edn. Approved Standard. NCCLS document M7-45. Wayne, PA: National Committee for Clinical Laboratory Standards.

Neeman, R., Keler, N., Barzilai, A., Korenman, Z. \& Sela, S. (1998). Prevalence of internalisation-gene, prtF1, among group-A streptococcus strains isolated from asymptomatic carriers. Lancet 352, 19741977.

Ozeri, V., Rosenshine, I., Mosher, D. F., Fassler, R. \& Hanski, E. (1998). Roles of integrins and fibronectin in the entry of Streptococcus pyogenes into cells via protein F1. Mol Microbiol 30, 625-637.

Pérez-Trallero, E., García-de-la-Fuente, C., García-Rey, C., Baquero, F., Aguilar, L., Dal-Ré, R., García-de-Lomas, J. \& Spanish Surveillance Group for Respiratory Pathogens (2005). Geographical and ecological analysis of resistance, coresistance, and coupled resistance to antimicrobials in respiratory pathogenic bacteria in Spain. Antimicrob Agents Chemother 49, 1965-1972.

Schrager, H. M., Rheinwald, J. G. \& Wessels, M. R. (1996). Hyaluronic acid capsule and the role of streptococcal entry into keratinocytes in invasive skin infection. J Clin Invest 98, 1954-1958.

Schrager, H. M., Albertí, S., Cywes, C., Dougherty, G. J. \& Wessels, M. R. (1998). Hyaluronic acid capsule modulates M-protein mediated adherence and acts as a ligand for attachment of group A Streptococcus to CD44 on human keratinocytes. J Clin Invest 101, 1708-1716.

Seppala, H., Nissinen, A., Yu, Q. \& Huovinen, P. (1993). Three different phenotypes of erythromycin-resistant Streptococcus pyogenes in Finland. J Antimicrob Chemother 32, 885-891.

Seppala, H., Skurnik, M., Soini, H., Roberts, M. C. \& Huovinen, P. (1998). A novel erythromycin resistance methylase gene (ermTR) in Streptococcus pyogenes. Antimicrob Agents Chemother 42, 257-262.

Spinaci, C., Magi, G., Zampaloni, C., Vitali, L. A., Paoletti, C., Catania, M. R., Prenna, M., Ferrante, L., Ripa, S. \& other authors (2004). Genetic diversity of cell-invasive erythromycin-resistant and -susceptible group A streptococci determined by analysis of the $R D 2$ region of the prtF1 gene. J Clin Microbiol 42, 639-644.

Spinaci, C., Magi, G. E. V. P. \& Facinelli, B. (2006). Persistence of erythromycin-resistant group A streptococci in cultured respiratory cells. Pediatr Infect Dis J 25, 880-883.

Sutcliffe, J., Grebe, T., Tait-Kamradt, A. \& Wondrack, L. (1996). Detection of erythromycin-resistant determinants by PCR. Antimicrob Agents Chemother 40, 2562-2566.

Tsai, P.-J., Lin, Y.-S., Kuo, C.-F., Lei, H.-Y. \& Wu, J.-J. (1999). Group A streptococcus induces apoptosis in human epithelial cells. Infect Immun 67, 4334-4339. 